\title{
Jacobi Theta Functions over Number Fields
}

\author{
By \\ Olav K. Richter ${ }^{1}$ and Howard Skogman ${ }^{2}$ \\ ${ }^{1}$ University of North Texas, Denton, TX, USA \\ ${ }^{2}$ State University of New York, Brockport, NY, USA \\ Received August 26, 2002 \\ Published online August 11, 2003 (C) Springer-Verlag 2003
}

\begin{abstract}
We use Jacobi theta functions to construct examples of Jacobi forms over number fields. We determine the behavior under modular transformations by regarding certain coefficients of the Jacobi theta functions as specializations of symplectic theta functions. In addition, we show how sums of those Jacobi theta functions appear as a single coefficient of a symplectic theta function.
\end{abstract}

2000 Mathematics Subject Classification: 11F55; 11F27, 11F41

Key words: Jacobi forms, theta functions

\section{Introduction}

Eichler and Zagier [2] develop a theory of holomorphic Jacobi forms and show that Jacobi theta functions corresponding to positive definite quadratic forms are examples of such forms. Skoruppa [14] introduces skew-holomorphic Jacobi forms and presents examples using Jacobi theta functions corresponding to indefinite quadratic forms with signature $(1, n-1)$. In this paper, we use Jacobi theta functions to create examples of Jacobi forms and skew-holomorphic Jacobi forms over number fields. More precisely, we define $\Theta_{Q, R, w}^{(K)}(\tau, z)$, a Jacobi theta function attached to an arbitrary quadratic form defined over a number field $K$. We present two different methods to determine the behavior of $\Theta_{Q, R, w}^{(K)}(\tau, z)$ under modular transformations.

The first approach is based on Eichler's "embedding trick" (see Eichler [1], for example). Friedberg [3] and Richter [8] prove transformation laws for $\vartheta\left(Z,\left(\begin{array}{l}r \\ s\end{array}\right), w, f\right)$, a modified version of the usual symplectic theta function. We proceed as in [8] and regard certain coefficients of $\Theta_{Q, R, w}^{(K)}(\tau, z)$ as specializations of $\vartheta\left(Z,\left(\begin{array}{l}0 \\ 0\end{array}\right), w, f\right)$. As an immediate consequence, we obtain the transformation law of $\Theta_{Q, R, w}^{(K)}(\tau, z)$ under modular transformations. A similar idea has been used in the literature to construct modular forms over number fields using theta functions (see for example, Stark [16], Imamoḡlu [5] and [6], Richter [9], [10], and [11]).

In the second approach, we follow Skogman [12] and [13] and present a different construction of Jacobi forms over number fields. We show how sums of $\Theta_{Q, R, w}^{(K)}(\tau, z)$ appear as a single coefficient of $\vartheta\left(Z,\left(\begin{array}{l}0 \\ 0\end{array}\right), w, 0\right)$, which determines 
how these sums behave under modular transformations. This is a very natural construction: In analogy, Jacobi forms (over $\mathbb{Q}$ ) appear as Fourier coefficients of Siegel modular forms (see Eichler and Zagier [2]).

\section{Notation and Terminology}

Let $K$ be an algebraic number field with $r_{1}$ real conjugates and $r_{2}$ pairs of complex conjugates. The real conjugates of an element $a$ in $K$ are denoted by $a^{(1)}, \ldots, a^{\left(r_{1}\right)}$ and the complex conjugates by $a^{\left(r_{1}+1\right)}, \ldots, a^{\left(r_{1}+2 r_{2}\right)}$, where $a^{\left(j+r_{2}\right)}=$ $\overline{a^{(j)}}$ for $r_{1}+1 \leqslant j \leqslant r_{1}+r_{2}$. Let $\delta_{K}$ be the different of $K$, and $\mathfrak{D}_{K}$ be the ring of integers of $K$, and set $\Gamma=\mathrm{SL}_{2}\left(\mathfrak{D}_{K}\right)$. The Jacobi group of $K$ is given by

$$
\Gamma^{J}(K)=\Gamma \ltimes \mathfrak{D}_{K}^{2} .
$$

Denote the field of complex numbers by $\mathbb{C}$ and let $\mathbb{U}$ be the usual upper half plane. Let $\mathscr{Q}=\left\{u+v k \mid u, v \in \mathbb{C}, k^{2}=-1, a k=k \bar{a}, \forall a \in \mathbb{C}\right\}$ be the full ring of quaternions and $\mathbb{H}_{\mathscr{Q}}=\left\{x+y k \in \mathscr{Q} \mid x \in \mathbb{C}, y \in \mathbb{R}^{+}\right\}$be the quaternionic upper half plane consisting of quaternions with no $j$-component and positive $k$-component. Set $\mathscr{Z}=\mathbb{C}^{r_{1}} \mathscr{Q}^{r_{2}}$. We write a typical element $z \in \mathscr{Z}$ as $z=\left(z_{1}, \ldots, z_{r_{1}+r_{2}}\right)$, where $z_{j} \in \mathbb{C}$ for $j=1, \ldots, r_{1}$, and $z_{j}=u_{j}+v_{j} k \in \mathscr{Q}$ for $j=r_{1}+1, \ldots, r_{1}+r_{2}$. The corresponding upper half space is $\mathfrak{H}=\llbracket^{r_{1}} \llbracket_{0}^{r_{2}}$ and we write a typical element as $\tau=$ $\left(\tau_{1}, \ldots, \tau_{r_{1}+r_{2}}\right) \in \mathfrak{H}$ where $\tau_{j}=x_{j}+y_{j} \in \mathbb{U}$ for $j=1, \ldots, r_{1}$, and $\tau_{j}=x_{j}+y_{j} k \in \mathbb{Q}_{\mathscr{Q}}$ for $j=r_{1}+1, \ldots, r_{1}+r_{2}$. We have $\bar{\tau}=\left(\overline{\tau_{1}}, \ldots, \overline{\tau_{r_{1}+r_{2}}}\right)$ where, as usual, $\overline{\tau_{j}}=x_{j}-$ $i y_{j}$ for $j=1, \ldots, r_{1}$ and $\overline{\tau_{j}}=\overline{x_{j}}-y_{j} k$ for $j=r_{1}+1, \ldots, r_{1}+r_{2}$. A matrix $\left(\begin{array}{ll}\alpha & \beta \\ \gamma & \delta\end{array}\right) \in \Gamma$ acts on $\tau \in \mathfrak{H}$ by

$$
\left(\begin{array}{ll}
\alpha & \beta \\
\gamma & \delta
\end{array}\right) \circ \tau=\left(\left(\begin{array}{cc}
\alpha & \beta \\
\gamma & \delta
\end{array}\right)^{(1)} \circ \tau_{1}, \ldots,\left(\begin{array}{cc}
\alpha & \beta \\
\gamma & \delta
\end{array}\right)^{\left(r_{1}+r_{2}\right)} \circ \tau_{r_{1}+r_{2}}\right)
$$

where

$$
\left(\begin{array}{ll}
\alpha & \beta \\
\gamma & \delta
\end{array}\right)^{(j)} \circ \tau_{j}=\left(\alpha^{(j)} \tau_{j}+\beta^{(j)}\right)\left(\gamma^{(j)} \tau_{j}+\delta^{(j)}\right)^{-1}
$$

An element $\left(\left(\begin{array}{ll}\alpha & \beta \\ \gamma & \delta\end{array}\right),(\lambda, \mu)\right) \in \Gamma^{J}(K)$ acts on $(\tau, z) \in \mathfrak{H} \times \mathscr{Z}$ by

$$
\begin{aligned}
& \left(\begin{array}{ll}
\alpha & \beta \\
\gamma & \delta
\end{array}\right) \circ(\tau, z) \\
& =\left(\left(\begin{array}{ll}
\alpha & \beta \\
\gamma & \delta
\end{array}\right)^{(1)} \circ \tau_{1}, \ldots,\left(\begin{array}{ll}
\alpha & \beta \\
\gamma & \delta
\end{array}\right)^{\left(r_{1}+r_{2}\right)} \circ \tau_{r_{1}+r_{2}},\left(\gamma^{(1)} \tau_{1}+\delta^{(1)}\right)^{-1} z_{1}, \ldots\right. \\
& \left.\ldots,\left(\gamma^{\left(r_{1}+r_{2}\right)} \tau_{r_{1}+r_{2}}+\delta^{\left(r_{1}+r_{2}\right)}\right)^{-1} z_{r_{1}+r_{2}}\right)
\end{aligned}
$$

and

$$
\begin{aligned}
(\lambda, \mu) \circ(\tau, z)= & \left(\tau_{1}, \ldots, \tau_{r_{1}+r_{2}}, z_{1}+\tau_{1} \lambda^{(1)}+\mu^{(1)}, \ldots\right. \\
& \left.\ldots, z_{r_{1}+r_{2}}+\tau_{r_{1}+r_{2}} \lambda^{\left(r_{1}+r_{2}\right)}+\mu^{\left(r_{1}+r_{2}\right)}\right) .
\end{aligned}
$$


As in the classical case (see Eichler and Zagier [2]), (2) and (3) jointly define a group action of $\Gamma^{J}(K)$ on $\mathfrak{S} \times \mathscr{Z}$.

Furthermore, for $\gamma$ and $\delta$ in $K$ and $\tau$ in $\mathfrak{H}$, we define

$$
\mathscr{N}(\gamma \tau+\delta)=\prod_{j=1}^{r_{1}}\left(\gamma^{(j)} \tau_{j}+\delta^{(j)}\right) \prod_{j=r_{1}+1}^{r_{1}+r_{2}}\left\|\gamma^{(j)} \tau_{j}+\delta^{(j)}\right\|^{2}
$$

where $\left\|\gamma^{(j)} \tau_{j}+\delta^{(j)}\right\|^{2}=\left|\gamma^{(j)} x_{j}+\delta^{(j)}\right|^{2}+\left|\gamma^{(j)}\right|^{2} y_{j}^{2}$ is the usual norm of the quaternion. In particular,

$$
\mathscr{N}(\gamma \tau+\delta)^{1 / 2}=\prod_{j=1}^{r_{1}}\left(\gamma^{(j)} \tau_{j}+\delta^{(j)}\right)^{1 / 2} \prod_{j=r_{1}+1}^{r_{1}+r_{2}}\left\|\gamma^{(j)} \tau_{j}+\delta^{(j)}\right\|,
$$

where each of the $r_{1}$ square roots on the right is given by the principal value.

For $u+v k \in \mathscr{Q}$, define $\|u+v k\|_{\mathbb{C}}=u+i v$ and $\|u+v k\|_{\overline{\mathbb{C}}}=\bar{u}+i \bar{v}$. Let $m={ }^{t}\left(m_{1}, \ldots,{ }^{t} m_{r_{1}+2 r_{2}}\right)$, where $m_{1}, \ldots, m_{r_{1}+2 r_{2}}$ are vectors in $\mathbb{C}^{l}$ (for some $l \in \mathbb{N}$ ), such that $m_{j+r_{2}}=\overline{m_{j}}$ for $j=r_{1}+1, \ldots, r_{1}+r_{2}$. Furthermore, set $\widetilde{m}_{j}={ }^{t} m_{j} m_{j}$ and $\widetilde{m}={ }^{t} m m$. For, $\gamma, \delta, \lambda$ in $K$, and $(\tau, z) \in \mathfrak{H} \times \mathscr{Z}$, it will be useful to define

$$
\begin{aligned}
& \mathscr{T}_{\mathscr{R}}\left[{ }^{t} m z(\gamma \tau+\delta)^{-1} \gamma z m\right] \\
& =\sum_{j=1}^{r_{1}} \widetilde{m}_{j} \frac{\gamma^{(j)}\left(z_{j}\right)^{2}}{\gamma^{(j)} \tau_{j}+\delta^{(j)}} \\
& \quad+\sum_{j=r_{1}+1}^{r_{1}+r_{2}}\left\|m_{j}\left(u_{j}+\overline{v_{j}} k\right)\left(\gamma^{(j)} \tau_{j}+\delta^{(j)}\right)^{-1} \gamma^{(j)}\left(u_{j}+v_{j} k\right) m_{j}\right\|_{\mathbb{C}} \\
& \quad+\sum_{j=r_{1}+1}^{r_{1}+r_{2}}\left\|m_{j}\left(u_{j}+\overline{v_{j}} k\right)\left(\gamma^{(j)} \tau_{j}+\delta^{(j)}\right)^{-1} \gamma^{(j)}\left(u_{j}+v_{j} k\right) m_{j}\right\|_{\overline{\mathbb{C}}},
\end{aligned}
$$

and

$$
\begin{aligned}
\mathscr{T}_{\mathscr{R}}\left[{ }^{t} m(\lambda \tau \lambda+2 \lambda z) m\right]= & \sum_{j=1}^{r_{1}} \widetilde{m}_{j}\left(\lambda^{(j)^{2}} \tau_{j}+2 \lambda^{(j)} z_{j}\right) \\
& +\sum_{j=r_{1}+1}^{r_{1}+r_{2}}\left\|m_{j}\left(\lambda^{(j)} \tau_{j} \lambda^{(j)}+2 \lambda^{(j)} z_{j}\right) m_{j}\right\|_{\mathbb{C}} \\
& \left.+\sum_{j=r_{1}+1}^{r_{1}+r_{2}} \| m_{j} m^{(j)} \tau_{j} \lambda^{(j)}+2 \lambda^{(j)} z_{j}\right) m_{j} \|_{\mathbb{C}} .
\end{aligned}
$$

Note that if $K=\mathbb{Q}$, then

$$
\mathscr{T}_{\mathscr{R}}\left[{ }^{t} m z(\gamma \tau+\delta)^{-1} \gamma z m\right]=\frac{\widetilde{m} \gamma z^{2}}{\gamma \tau+\delta}, \quad \mathscr{T}_{\mathscr{R}}\left[{ }^{t} m(\lambda \tau \lambda+2 \lambda z) m\right]=\widetilde{m}\left(\lambda^{2} \tau+2 \lambda z\right) .
$$


We will construct functions $f: \mathfrak{H} \times \mathscr{Z} \rightarrow \mathbb{C}$ which satisfy the following transformation properties: For $\left(\left(\begin{array}{ll}\alpha & \beta \\ \gamma & \delta\end{array}\right),(\lambda, \mu)\right) \in \Gamma^{J}(K)$,

$$
\begin{aligned}
f\left(\left(\begin{array}{ll}
\alpha & \beta \\
\gamma & \delta
\end{array}\right) \circ(\tau, z)\right)= & \chi\left(\left(\begin{array}{ll}
\alpha & \beta \\
\gamma & \delta
\end{array}\right)\right) \mathscr{N}(\gamma \tau+\delta)^{k_{1}} \cdot \mathcal{N}(\overline{\gamma \tau+\delta})^{k_{2}} \\
& \times \exp \left\{2 \pi i \mathscr{T}_{\mathscr{R}}\left[m z(\gamma \tau+\delta)^{-1} \gamma z m\right]\right\} f(\tau, z)
\end{aligned}
$$

and

$$
f((\lambda, \mu) \circ(\tau, z))=\exp \left\{-2 \pi i \mathscr{T}_{\mathscr{R}}\left[{ }^{t} m(\lambda \tau \lambda+2 \lambda z) m\right]\right\} f(\tau, z),
$$

where $\chi\left(\left(\begin{array}{ll}\alpha & \beta \\ \gamma & \delta\end{array}\right)\right)$ is a root of unity, and $m$ as above. Obviously, equations (4) and (5) generalize the transformation laws for Jacobi forms and skew-holomorphic Jacobi forms. Hence functions $f: \mathfrak{H} \times \mathscr{Z} \rightarrow \mathbb{C}$ satisfying (4) and (5) are generalizations of Jacobi forms and skew-holomorphic Jacobi forms.

\section{Symplectic Theta Function}

It will be useful to define $U[V]={ }^{t} V U V$ for any vector or matrix $V$ and any matrix $U$. The symplectic group,

$$
\operatorname{Sp}_{n}(\mathbb{R})=\left\{M=\left(\begin{array}{ll}
A & B \\
C & D
\end{array}\right) \mid M \in \mathrm{M}_{2 n, 2 n}(\mathbb{R}) \text { such that } J[M]=J=\left(\begin{array}{cc}
0 & -I_{n} \\
I_{n} & 0
\end{array}\right)\right\}
$$

where $I_{n}$ is the $n \times n$ identity matrix, acts on the Siegel upper half plane

$$
\mathfrak{S}^{(n)}=\left\{Z \in \mathrm{M}_{n, n}(\mathbb{C}) \mid Z={ }^{t} Z \text { and } \operatorname{Im}(Z)>0\right\} .
$$

The action of $M$ on $Z$ is given by

$$
M \circ Z=(A Z+B)(C Z+D)^{-1} .
$$

Let $\Gamma^{(n)}=\operatorname{Sp}_{n}(\mathbb{Z})$. The theta subgroup

$$
\Gamma_{\vartheta}^{(n)}=\left\{\left(\begin{array}{cc}
A & B \\
C & D
\end{array}\right) \in \Gamma^{(n)} \mid A^{t} B, C^{t} D \text { have even diagonal entries }\right\},
$$

acts on the symplectic theta function,

$$
\vartheta\left(Z,\left(\begin{array}{l}
r \\
s
\end{array}\right)\right)=\sum_{m \in \mathbb{Z}^{n}} \exp \left\{\pi i\left(Z[m+s]-2^{t} m r-{ }^{t} s r\right)\right\},
$$

where $r$ and $s$ are column vectors in $\mathbb{C}^{n}$. It is well known (see Eichler [1], for example) that for $M=\left(\begin{array}{cc}A & B \\ C & D\end{array}\right) \in \Gamma_{\vartheta}^{(n)}$,

$$
\vartheta\left(M \circ Z, M\left(\begin{array}{l}
r \\
s
\end{array}\right)\right)=\chi(M)\{\operatorname{det}(C Z+D)\}^{1 / 2} \vartheta\left(Z,\left(\begin{array}{l}
r \\
s
\end{array}\right)\right)
$$

where $\chi(M)$ is an eighth root of unity which depends upon the chosen square root of $\operatorname{det}(C Z+D)$, but which is otherwise independent of $Z, r$, and $s$. Stark [15] 
determines $\chi(M)$ in the important special case that both $C$ and $D$ are nonsingular and that $p D^{-1}$ is integral for some odd prime $p$. The main result in [15] is

Theorem 1. Suppose $M=\left(\begin{array}{ll}A & B \\ C & D\end{array}\right)$ is in $\Gamma_{\vartheta}^{(n)}$ where $C^{-1}$ and $D^{-1}$ exist. Suppose further that for some odd prime $p, p D^{-1}$ is integral. Then $(\bmod p)$, the symmetric matrix $p D^{-1} C$ has rank $h$ where $\operatorname{det}(D)= \pm p^{h}$. Let $\left(p D^{-1} C\right)^{(h)}$ be a nonsingular $(\bmod p) h \times h$ principal submatrix of $p D^{-1} C$ and $\sigma$ be the signature (the number of positive eigenvalues minus the number of negative eigenvalues) of $C^{-1} D$. Then

$$
\begin{aligned}
& \chi(M)\{\operatorname{det}(C Z+D)\}^{1 / 2} \\
& =\varepsilon_{p}^{-h}\left(\frac{2^{h} \operatorname{det}\left[\left(p D^{-1} C\right)^{(h)}\right]}{p}\right) e^{\frac{\pi i \sigma}{4}}|\operatorname{det}(C)|^{1 / 2}\left\{\operatorname{det}\left[-i C^{-1}(C Z+D)\right]\right\}^{1 / 2},
\end{aligned}
$$

where $\varepsilon_{p}=1$ for $p \equiv 1 \bmod 4, \varepsilon_{p}=i$ for $p \equiv 3 \bmod 4,(\dot{\bar{p}})$ is the Legendre symbol, $|\operatorname{det}(C)|^{1 / 2}$ is positive and $\left\{\operatorname{det}\left[-i C^{-1}(C Z+D)\right]\right\}^{1 / 2}$ is given by analytic continuation from the principal value when $Z=-C^{-1} D+i Y$. Alternatively, if just $C^{-1}$ exists and $p C^{-1}$ is integral, $\operatorname{det}(C)= \pm p^{h}$, then $p C^{-1} D(\bmod p)$ has rank $h$ and

$$
\begin{aligned}
& \chi(M)\{\operatorname{det}(C Z+D)\}^{1 / 2} \\
& =\varepsilon_{p}^{-h}\left(\frac{-2}{p}\right)^{h}\left(\frac{\operatorname{det}\left[\left(p C^{-1} D\right)^{(h)}\right]}{p}\right)|\operatorname{det}(C)|^{1 / 2}\left\{\operatorname{det}\left[-i C^{-1}(C Z+D)\right]\right\}^{1 / 2} .
\end{aligned}
$$

For $w \in \mathbb{C}^{n}, f$ a nonnegative integer, and $Z, r$, and $s$ as above, define

$$
\begin{aligned}
& \vartheta\left(Z,\left(\begin{array}{l}
r \\
s
\end{array}\right), w, f\right) \\
& \quad=\sum_{m \in \mathbb{Z}^{n}}\left({ }^{t} w(m+s)\right)^{f} \exp \left\{\pi i\left(Z[m+s]-2^{t} m r-{ }^{t} s r\right)\right\} .
\end{aligned}
$$

Note that for $f=0$ the theta functions in (6) and (8) coincide. Friedberg [3] and Richter [8] examine the transformation properties of $\vartheta\left(Z,\left(\begin{array}{l}r \\ s\end{array}\right), w, f\right)$ under modular transformations, and Richter [8] shows the following theorem:

Theorem 2. Let $M=\left(\begin{array}{cc}A & B \\ C & D\end{array}\right) \in \Gamma_{\vartheta}^{(n)}$. Then

$$
\begin{aligned}
\vartheta(M \circ & \left.Z, M\left(\begin{array}{l}
r \\
s
\end{array}\right),{ }^{t}(C Z+D)^{-1} w, f\right) \\
= & \chi(M)\{\operatorname{det}(C Z+D)\}^{1 / 2} \sum_{l=0}^{\left[\frac{[}{2}\right]} \frac{f !}{l !(f-2 l) !}(\pi i)^{-l} 2^{-2 l} \\
& \times\left(\left((C Z+D)^{-1} C\right)[w]\right)^{l} \vartheta\left(Z,\left(\begin{array}{l}
r \\
s
\end{array}\right), w, f-2 l\right),
\end{aligned}
$$

where $\chi(M)$ is as in (7). 
Remark. Friedberg [3] proves Theorem 2 (phrased slightly differently) in the special case where $\left((C Z+D)^{-1} C\right)[w]=0$, in which case the right hand side in (9) reduces to $\chi(M)\{\operatorname{det}(C Z+D)\}^{1 / 2} \vartheta\left(Z,\left(\begin{array}{l}r \\ s\end{array}\right), w, f\right)$.

In the next section, we define $\theta_{Q, R, w}^{(K)}(\tau, z)$, a theta function over a number field $K$, and we will use Theorem 2 to determine the behavior of $\theta_{Q, R, w}^{(K)}(\tau, z)$ under modular transformations.

\section{Theta Functions of Quadratic Forms}

Let $K$ be an algebraic number field with $r_{1}$ real conjugates and $r_{2}$ pairs of complex conjugates. We use the notation introduced in section 2 . Also, we write $U[V]={ }^{t} V U V$ (as before) and $U\{V\}={ }^{t} V U \bar{V}$ for any vector or matrix $V$ and any matrix $U$. Let $Q$ be a symmetric $n \times n$ matrix with entries in $\mathfrak{D}_{K}$ defining the quadratic form $Q[x]$, where $x \in \mathbb{C}^{n}$. If, in addition, $Q$ has diagonal entries which are divisible by 2 , we say that $Q$ is of level $\mathfrak{N}\left(\mathfrak{N}\right.$ an ideal in $\mathfrak{D}_{K}$ ) whenever the following two conditions are satisfied:

a) The matrix $N Q^{-1}$ has entries in $\mathfrak{D}_{K}$ and 2 divides the diagonal entries of $N Q^{-1}$ for all $N \in \mathfrak{N}$.

b) If $\mathfrak{M}$ is any integral ideal satisfying a), i.e. $M Q^{-1}$ has entries in $\mathfrak{D}_{K}$ and 2 divides the diagonal entries of $M Q^{-1}$ for all $M \in \mathfrak{M}$, then $\mathfrak{N}$ divides $\mathfrak{M}$.

Suppose that all of the real conjugates of $Q$ are of the same type $(p, q)$. Hence there exist matrices $L_{j}$ in $G L_{n}(\mathbb{R})$ such that

$$
Q^{(j)}={ }^{t} L_{j} E_{p, q} L_{j}, \quad j=1, \ldots, r_{1}
$$

and there exist matrices $L_{j}$ in $G L_{n}(\mathbb{C})$ such that

$$
Q^{(j)}={ }^{t} L_{j} L_{j}, \quad j=r_{1}+1, \ldots, r_{1}+r_{2},
$$

where $E_{p, q}=\left(\begin{array}{ll}I_{p} & \\ & -I_{q}\end{array}\right)$, and $I_{p}$ and $I_{q}$ are the $p \times p$ and $q \times q$ identity matrices, respectively. We set

$$
R_{j}={ }^{t} L_{j} \overline{L_{j}}
$$

For all $j, R_{j}$ is a majorant of $Q^{(j)}$, i.e.

$$
\overline{R_{j}} Q^{(j)}{ }^{-1} R_{j}=\overline{Q^{(j)}} \quad \text { and } \quad{ }^{t} R_{j}=\overline{R_{j}}>0 .
$$

We define a theta function corresponding to an arbitrary quadratic form by

Definition 1. Let $Q$ be a symmetric $n \times n$ matrix with entries in $\mathfrak{D}_{K}$ such that 2 divides the diagonal entries of $Q$ and such that $Q$ is of level $\mathfrak{N}\left(\mathfrak{M}\right.$ an ideal in $\left.\mathfrak{D}_{K}\right)$. Assume that all of the real conjugates $Q^{(j)}$ of $Q$ are of the same type $(p, q)$ and set $L_{j}$ as in (10) and (11) and $R_{j}$ as in (12). Let $w={ }^{t}\left({ }^{t} w_{1}, \ldots,{ }^{t} w_{r_{1}+2 r_{2}}\right)$, where $w_{1}, \ldots, w_{r_{1}+2 r_{2}}$ are vectors in $\mathbb{C}^{n}$ such that $w_{j+r_{2}}=\overline{w_{j}}$ for $j=r_{1}+1, \ldots, r_{1}+r_{2}$. Let $f$ be a nonnegative rational integer. For an ideal $\mathfrak{I} \subset \mathfrak{D}_{K}$ and for 
$(\tau, z) \in \mathfrak{H} \times \mathscr{Z}=\mathbb{H}^{r_{1}} \mathbb{T}_{\mathscr{Q}}^{r_{2}} \times \mathbb{C}^{r_{1}} \mathscr{Q}^{r_{2}}$, we set

$$
\begin{aligned}
\theta_{Q, R, w, f}^{(K)}(\tau, z) & =\sum_{\kappa \in \mathfrak{J}^{n}}\left(\sum_{j=1}^{r_{1}}{ }^{t} \kappa^{(j)} Q^{(j)} w_{j} z_{j}\right. \\
& \left.+\sum_{j=r_{1}+1}^{r_{1}+r_{2}}\left\|t^{(j)}{ }^{t} L_{j} z_{j} L_{j} w_{j}\right\|_{\mathbb{C}}+\left\|{ }^{t} \kappa^{(j) t} L_{j} z_{j} L_{j} w_{j}\right\|_{\overline{\mathbb{C}}}\right)^{f} \\
& \times \exp \pi i\left(\sum_{j=1}^{r_{1}} Q^{(j)}\left[\kappa^{(j)}\right] x_{j}+i R_{j}\left[\kappa^{(j)}\right] y_{j}\right. \\
& \left.\left.+\sum_{j=r_{1}+1}^{r_{1}+r_{2}}\left\|{ }^{t} \kappa^{(j) t} L_{j} \tau_{j} L_{j} \kappa^{(j)}\right\|_{\mathbb{C}}+\left\|{ }^{t} \kappa^{(j)}{ }^{t} L_{j} \tau_{j} L_{j} \kappa^{(j)}\right\|_{\overline{\mathbb{C}}}\right)\right\},
\end{aligned}
$$

where $\kappa={ }^{t}\left(\kappa_{1}, \ldots, \kappa_{n}\right)$ and $\kappa^{(j)}={ }^{t}\left(\kappa_{1}^{(j)}, \ldots, \kappa_{n}^{(j)}\right)$. Furthermore, we define:

$$
\begin{aligned}
& \Theta_{Q, R, w}^{(K)}(\tau, z) \\
& =\sum_{f \geqslant 0} \frac{(2 \pi i)^{2 f}}{(2 f) !} \theta_{Q, R, w, 2 f}^{(K)}(\tau, z) \\
& =\sum_{\kappa \in \mathfrak{J}^{n}} \exp \left\{\pi i \left(\sum_{j=1}^{r_{1}} Q^{(j)}\left[\kappa^{(j)}\right] x_{j}+i R_{j}\left[\kappa^{(j)}\right] y_{j}+2{ }^{t} \kappa^{(j)} Q^{(j)} w_{j} z_{j}\right.\right. \\
& \quad+\sum_{j=r_{1}+1}^{r_{1}+r_{2}}\left\|t \kappa^{(j)}{ }^{t} L_{j} \tau_{j} L_{j} \kappa^{(j)}+2{ }^{t} \kappa^{(j) t} L_{j} z_{j} L_{j} w_{j}\right\|_{\mathbb{C}} \\
& \left.\left.\quad+\sum_{j=r_{1}+1}^{r_{1}+r_{2}}\left\|t^{(j)}{ }^{t} L_{j} \tau_{j} L_{j} \kappa^{(j)}+2{ }^{t} \kappa^{(j)}{ }^{t} L_{j} z_{j} L_{j} w_{j}\right\|_{\overline{\mathbb{C}}}\right)\right\} .
\end{aligned}
$$

Remarks. a) If $K=\mathbb{Q}$, then $\Theta_{Q, R, w}^{(\mathbb{Q})}(\tau, z)$ is the usual Jacobi theta function. If, in addition, $Q$ is of type $(1, n-1)$, then $\Theta_{Q, R, w}^{(\mathbb{Q})}(\tau, z)$ is a skew-holomorphic Jacobi form in the sense of Skoruppa [14]. [13].

b) The theta function $\Theta_{Q, R, w}^{(K)}(\tau, z)$ generalizes the theta functions in [12] and

c) If $f$ is odd, then $\theta_{Q, R, w, f}^{(K)}(\tau, z)$ is identically zero.

d) For $\eta \in \mathfrak{D}_{K}, \theta_{Q, R, w, f}^{(K)}(\tau, z)$ (and hence also $\Theta_{Q, R, w}^{(K)}(\tau, z)$ ) is invariant under linear transformations, i.e.

$$
\theta_{Q, R, w, f}^{(K)}(\tau+\eta, z)=\theta_{Q, R, w, f}^{(K)}(\tau, z)
$$

Set

$$
L=\left(\begin{array}{ccc}
L_{1} & & \\
& \ddots & \\
& & L_{\operatorname{deg} K}
\end{array}\right)
$$


where $L_{j+r_{2}}=\overline{L_{j}}$ for $j=r_{1}+1, \ldots, r_{1}+r_{2}$. We generalize and improve the main results in [12] and [13] by the following theorem:

Theorem 3. Let $w_{1} \in \mathfrak{J}^{n}$ such that $w_{j}=w_{1}^{(j)}$ for all $j$ and such that $Q^{(j)} w_{j}=$ $R_{j} w_{j}$ for $j=1, \ldots, r_{1}$. For $\left(\left(\begin{array}{ll}\alpha & \beta \\ \gamma & \delta\end{array}\right),(\lambda, \mu)\right) \in \Gamma_{0}\left(\mathfrak{J}^{2} \delta_{K} \mathfrak{M}\right) \ltimes \mathfrak{D}_{K}^{2}$ we have

$$
\begin{aligned}
\Theta_{Q, R, w}^{(K)}\left(\left(\begin{array}{cc}
\alpha & \beta \\
\gamma & \delta
\end{array}\right) \circ(\tau, z)\right)= & \chi\left(\left(\begin{array}{cc}
\alpha & \beta \\
\gamma & \delta
\end{array}\right), Q\right) \mathscr{N}(\gamma \tau+\delta)^{p / 2} \mathscr{N}(\overline{\gamma \tau+\delta})^{q / 2} \\
& \left.\times \exp \left\{\pi i \mathscr{T} \mathscr{R}^{t}(L w) z(\gamma \tau+\delta)^{-1} \gamma z L w\right]\right\} \Theta_{Q, R, w}^{(K)}(\tau, z),
\end{aligned}
$$

and

$$
\begin{aligned}
& \Theta_{Q, R, w}^{(K)}((\lambda, \mu) \circ(\tau, z)) \\
& \left.\quad=\exp \left\{-\pi i \mathscr{T}_{\mathscr{R}}{ }^{t}(L w)(\lambda \tau \lambda+2 \lambda z) L w\right]\right\} \Theta_{Q, R, w}^{(K)}(\tau, z),
\end{aligned}
$$

where $\chi\left(\left(\begin{array}{ll}\alpha & \beta \\ \gamma & \delta\end{array}\right), Q\right)$ is an eighth root of unity. If $\delta \gg 0$ is a first degree prime of norm $d$, then

$$
\chi\left(\left(\begin{array}{ll}
\alpha & \beta \\
\gamma & \delta
\end{array}\right), Q\right)=\varepsilon_{d}^{-n}\left(\frac{\left(d \delta^{-1} 2 \gamma\right)^{n} \operatorname{det}(Q)}{\delta}\right)
$$

where $\mathscr{N}(\delta)=d$, and $\varepsilon_{d}=1$ for $d \equiv 1 \bmod 4$ and $\varepsilon_{d}=i$ for $d \equiv 3 \bmod 4$ and $(\dot{\bar{\delta}})$ is the quadratic symbol.

Remark. We pointed out that $\Theta_{Q, R, w}^{(K)}(\tau, z)$ is invariant under linear transformations. From (17), it follows that for $\left(\begin{array}{ll}\alpha & \beta \\ \gamma & \delta\end{array}\right) \in \Gamma_{0}\left(\mathfrak{J}^{2} \delta_{K} \mathfrak{N}\right)$ and for all algebraic
integers $\eta$,

$$
\chi\left(\left(\begin{array}{cc}
\alpha & \beta \\
\gamma & \delta
\end{array}\right)\left(\begin{array}{cc}
1 & \eta \\
0 & 1
\end{array}\right), Q\right)=\chi\left(\left(\begin{array}{cc}
\alpha & \beta \\
\gamma & \delta
\end{array}\right), Q\right) .
$$

Note that $\left(\begin{array}{ll}\alpha & \beta \\ \gamma & \delta\end{array}\right) \in \Gamma_{0}\left(\mathfrak{J}^{2} \delta_{K} \mathfrak{N}\right)$ implies that $(\gamma, \delta)=1$ and by Dirichlet's primes in progression theorem for number fields (see Hecke [4], for example) the arithmetic progression $\{\gamma \eta+\delta\}_{\eta \in \mathfrak{D}_{k}}$ contains infinitely many totally positive first degree primes. Hence the eighth root of unity is determined explicitly by (20) after locating a totally positive first degree prime with positive odd norm in the arithmetic progression $\{\gamma \eta+\delta\}_{\eta \in \mathfrak{D}_{k}}$.

The following two subsections are devoted to proving (17) and (18).

4.1. The modular transformation. We use the method presented in Richter [11] (for more details, see also [9] and [10]): We regard $\theta_{Q, R, w, f}^{(K)}(\tau, z)$ as a specialization of $\vartheta\left(Z,\left(\begin{array}{l}0 \\ 0\end{array}\right), w, f\right)$ and then apply Theorem 2 , which will yield (17). 
Let $\mu_{1}, \ldots, \mu_{\operatorname{deg} K}$ be an integral basis of the ideal $\mathfrak{I} \subset \mathfrak{D}_{K}$ and define the vector $\mu^{(j)}=\left(\mu_{1}^{(j)}, \ldots, \mu_{\operatorname{deg} K}^{(j)}\right)$. We define the $n \times n \operatorname{deg} K$ matrix

$$
W_{j}=\left(\begin{array}{ccc}
\mu^{(j)} & & \\
& \ddots & \\
& & \mu^{(j)}
\end{array}\right)
$$

and the $n \operatorname{deg} K \times n \operatorname{deg} K$ matrix $W={ }^{t}\left({ }^{t} W_{1}, \ldots,{ }^{t} W_{\operatorname{deg} K}\right)$. Note that $W^{-1}$ has entries in $\mathfrak{J}^{-1} \delta_{K}^{-1}$.

Let $\tau=\left(\tau_{1}, \ldots, \tau_{r_{1}+r_{2}}\right) \in \mathfrak{H}$. For $j=1, \ldots, r_{1}$ we set

$$
Z_{j}^{*}=\left(\begin{array}{cc}
\tau_{j} I_{p} & \\
& -\bar{\tau}_{j} I_{q}
\end{array}\right)
$$

and for $j=r_{1}+1, \ldots, r_{1}+r_{2}$, we define the $n \times n$ matrices $X_{j}=x_{j} I_{n}, \overline{X_{j}}=\bar{x}_{j} I_{n}$, and $Y_{j}=y_{j} I_{n}$. We also define the $n \operatorname{deg} K \times n \operatorname{deg} K$ matrix

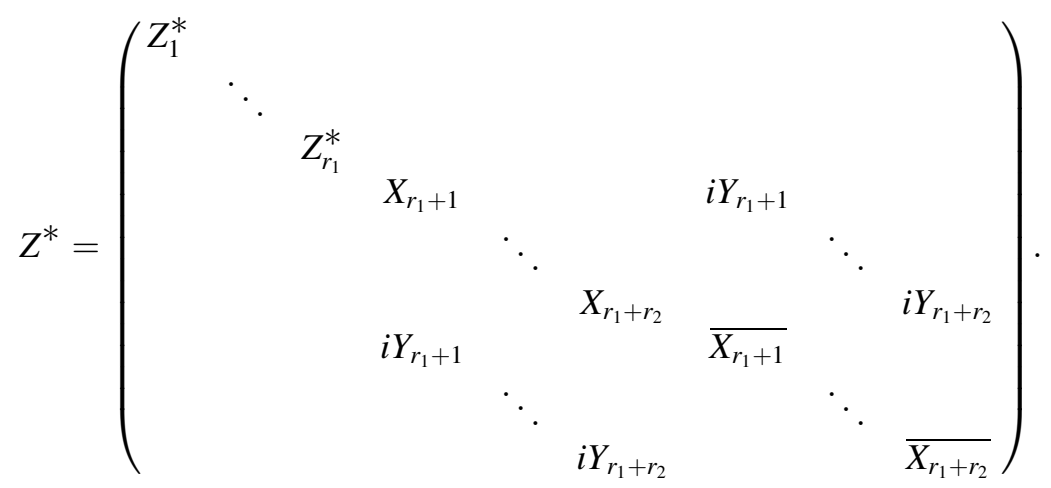

We set

$$
T=L W
$$

and

$$
Z={ }^{t} T Z^{*} T \text {. }
$$

It is not difficult to see that $Z \in \mathfrak{H}^{(n \operatorname{deg} K)}$ (see also [9] and [10]).

With $\widetilde{w}(z)={ }^{t} W^{t}\left(\widetilde{w}_{1}(z), \ldots,{ }^{\tau} \widetilde{w}_{r_{1}+2 r_{2}}(z)\right) \in \mathbb{C}^{n(\operatorname{deg} K)}$, where

$$
\widetilde{w}_{j}(z)= \begin{cases}z_{j} Q^{(j)} w_{j} & \text { for } j=1, \ldots, r_{1}, \\ \left\|L_{j} z_{j} L_{j} w_{j}\right\|_{\mathbb{C}} & \text { for } j=r_{1}+1, \ldots, r_{1}+r_{2}, \\ \left\|L_{j-r_{2}} z_{j-r_{2}} L_{j-r_{2}} w_{j-r_{2}}\right\|_{\overline{\mathbb{C}}} & \text { for } j=r_{1}+r_{2}+1, \ldots, r_{1}+2 r_{2},\end{cases}
$$

we have

$$
\theta_{Q, R, w, f}^{(K)}(\tau, z)=\vartheta\left(Z,\left(\begin{array}{l}
0 \\
0
\end{array}\right), \widetilde{w}(z), f\right) .
$$

In particular, (25) shows that $\theta_{Q, R, w, f}^{(K)}(\tau, z)$ and hence also $\Theta_{Q, R, w}^{(K)}(\tau, z)$ are not identically zero. 
To determine the behavior of $\theta_{Q, R, w, f}^{(K)}(\tau, z)$ under modular transformations, we want to apply Theorem 2 and therefore need a symplectic matrix $\left(\begin{array}{cc}A & B \\ C & D\end{array}\right)$ which expresses the action of $\left(\begin{array}{ll}\alpha & \beta \\ \gamma & \delta\end{array}\right) \in \Gamma$ on $Z$. For $\left(\begin{array}{ll}\alpha & \beta \\ \gamma & \delta\end{array}\right) \in \Gamma$, set

$$
\begin{aligned}
& A^{*}=\left(\begin{array}{ccc}
\alpha^{(1)} I_{n} & & \\
& \ddots & \\
& & \alpha^{(\operatorname{deg} K)} I_{n}
\end{array}\right), \quad D^{*}=\left(\begin{array}{ccc}
\delta^{(1)} I_{n} & & \\
& \ddots & \\
& & \delta^{(\operatorname{deg} K)} I_{n}
\end{array}\right) \text {, }
\end{aligned}
$$

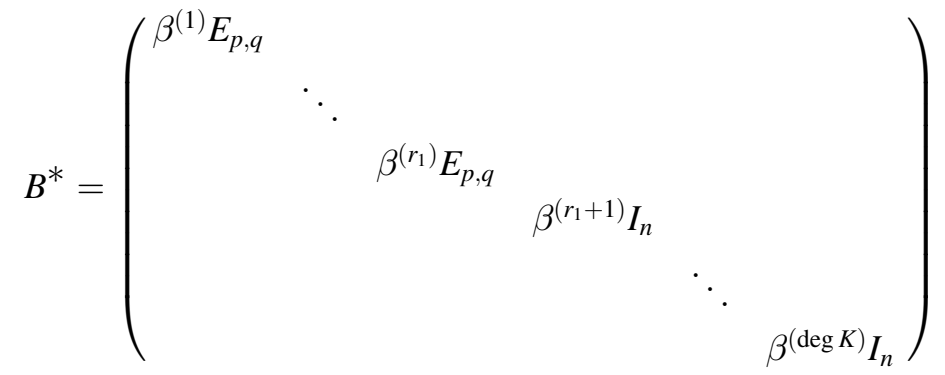

and

$$
C^{*}=\left(\begin{array}{cccccc}
\gamma^{(1)} E_{p, q} & & & & & \\
& \ddots & & & \\
& & \gamma^{\left(r_{1}\right)} E_{p, q} & & & \\
& & & \gamma^{\left(r_{1}+1\right)} I_{n} & & \\
& & & & \ddots & \\
& & & & & \gamma^{(\operatorname{deg} K)} I_{n}
\end{array}\right)
$$

Furthermore, we set

$$
\left(\begin{array}{ll}
A & B \\
C & D
\end{array}\right)=\left(\begin{array}{cc}
{ }^{t} T A^{*} T^{-1} & { }^{t} T B^{*} T \\
T^{-1} C^{*} T^{-1} & T^{-1} D^{*} T
\end{array}\right)
$$

It is easy to check that the diagram

$$
\begin{aligned}
\tau & \longrightarrow\left(\begin{array}{ll}
\alpha & \beta \\
\gamma & \delta
\end{array}\right) \circ \tau \\
\downarrow & \downarrow \\
Z^{*} & \longrightarrow\left(\begin{array}{ll}
A^{*} & B^{*} \\
C^{*} & D^{*}
\end{array}\right) \circ Z^{*}
\end{aligned}
$$

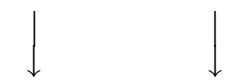

commutes. Hence

$$
\tau \mapsto\left(\begin{array}{cc}
\alpha & \beta \\
\gamma & \delta
\end{array}\right) \circ \tau \in \mathfrak{H}
$$


corresponds to

$$
Z \mapsto\left(\begin{array}{cc}
A & B \\
C & D
\end{array}\right) \circ Z \in \mathfrak{H}^{(n \operatorname{deg} K)}
$$

Also, it is easy to verify that

$$
\begin{aligned}
& { }^{t}(C Z+D)^{-1} \widetilde{w}(z) \\
& \quad={ }^{t} W \widetilde{w}\left(\left(\gamma^{(1)} \tau_{1}+\delta^{(1)}\right)^{-1} z_{1}, \ldots,\left(\gamma^{\left(r_{1}+r_{2}\right)} \tau_{r_{1}+r_{2}}+\delta^{\left(r_{1}+r_{2}\right)}\right)^{-1} z_{r_{1}+r_{2}}\right) .
\end{aligned}
$$

Hence

$$
\left(\left(\gamma^{(1)} \tau_{1}+\delta^{(1)}\right)^{-1} z_{1}, \ldots,\left(\gamma^{\left(r_{1}+r_{2}\right)} \tau_{r_{1}+r_{2}}+\delta^{\left(r_{1}+r_{2}\right)}\right)^{-1} z_{r_{1}+r_{2}}\right) \in \mathscr{Z}
$$

corresponds to

$$
{ }^{t}(C Z+D)^{-1} \widetilde{w}(z) \in \mathbb{C}^{n \operatorname{deg} K} .
$$

The entries of $A, B, C$ and $D$ are rational integers and $A^{t} B$ and $C^{t} D$ have even diagonal entries if $\gamma$ is in the ideal $\mathfrak{J}^{2} \delta_{K} N$ (see also [9] and [10]). Hence, for

$$
\left(\begin{array}{ll}
\alpha & \beta \\
\gamma & \delta
\end{array}\right) \in \Gamma_{0}\left(\mathfrak{I}^{2} \delta_{K} \mathfrak{N}\right)
$$

we have

$$
\left(\begin{array}{ll}
A & B \\
C & D
\end{array}\right) \in \Gamma_{\vartheta}^{(n \operatorname{deg} K)} .
$$

Straightforward computation shows that

$$
\begin{aligned}
\operatorname{det}(C Z+D) & =\prod_{j=1}^{r_{1}}\left(\gamma^{(j)} \tau_{j}+\delta^{(j)}\right)^{p}\left(\overline{\gamma^{(j)} \tau_{j}+\delta^{(j)}}\right)^{q} \prod_{j=r_{1}+1}^{r_{1}+r_{2}}\left\|\gamma^{(j)} \tau_{j}+\delta^{(j)}\right\|^{2 n} \\
& =\mathscr{N}(\gamma \tau+\delta)^{p} \mathscr{N}(\overline{\gamma \tau+\delta})^{q}
\end{aligned}
$$

and that

$$
\left.(C Z+D)^{-1} C[\widetilde{w}(z)]=\mathscr{T}_{\mathscr{R}}{ }^{t}(L w) z(\gamma \tau+\delta)^{-1} \gamma z L w\right]
$$

Hence by equations (9) and (25),

$$
\begin{aligned}
& \theta_{Q, R, w, f}^{(K)}\left(\left(\begin{array}{ll}
\alpha & \beta \\
\gamma & \delta
\end{array}\right) \circ(\tau, z)\right) \\
& =\chi\left(\left(\begin{array}{ll}
\alpha & \beta \\
\gamma & \delta
\end{array}\right), Q\right) \mathscr{N}(\gamma \tau+\delta)^{p / 2} \mathscr{N}(\overline{\gamma \tau+\delta})^{q / 2} \sum_{l=0}^{\left[\frac{f}{2}\right]} \frac{f !(\pi i)^{-l} 2^{-2 l}}{l !(f-2 l) !} \\
& \quad \times\left(\mathscr{T}_{\mathscr{R}}\left[(L w) z(\gamma \tau+\delta)^{-1} \gamma z L w\right]\right)^{l} \theta_{Q, R, w, f-2 l}^{(K)}(\tau, z)
\end{aligned}
$$


where $\chi\left(\left(\begin{array}{ll}\alpha & \beta \\ \gamma & \delta\end{array}\right), Q\right)$ is an eighth root of unity depending on $\left(\begin{array}{ll}\alpha & \beta \\ \gamma & \delta\end{array}\right)$ and $Q$. As an immediate consequence we obtain (17):

$$
\begin{aligned}
& \Theta_{Q, R, w}^{(K)}\left(\left(\begin{array}{ll}
\alpha & \beta \\
\gamma & \delta
\end{array}\right) \circ(\tau, z)\right) \\
&=\chi\left(\left(\begin{array}{ll}
\alpha & \beta \\
\gamma & \delta
\end{array}\right), Q\right) \mathscr{N}(\gamma \tau+\delta)^{p / 2} \mathscr{N}(\overline{\gamma \tau+\delta})^{q / 2} \\
& \times \sum_{f \geqslant 0} \sum_{l=0}^{f} \frac{\left.\left(\pi i \mathscr{\mathscr { T }}{ }^{t}(L w) z(\gamma \tau+\delta)^{-1} \gamma z(L w)\right]\right)^{l}}{l !} \frac{(2 \pi i)^{2 f-2 l}}{(2 f-2 l) !} \Theta_{Q, R, w, 2 f-2 l}^{(K)}(\tau, z) \\
&= \chi\left(\left(\begin{array}{ll}
\alpha & \beta \\
\gamma & \delta
\end{array}\right), Q\right) \mathscr{N}(\gamma \tau+\delta)^{p / 2} \mathscr{N}(\overline{\gamma \tau+\delta})^{q / 2} \\
&\left.\times \exp \left\{\pi i \mathscr{T}_{\mathscr{R}}{ }^{t}(L w) z(\gamma \tau+\delta)^{-1} \gamma z L w\right]\right\} \Theta_{Q, R, w}^{(K)}(\tau, z) .
\end{aligned}
$$

It remains to determine the eighth root of unity in (27). Suppose that $\left(\begin{array}{ll}\alpha & \beta \\ \gamma & \delta\end{array}\right) \in \Gamma_{0}\left(\mathfrak{J}^{2} \delta_{K} \mathfrak{M}\right)$, where $\delta \gg 0$ is a first degree prime in $\mathfrak{D}_{K}$ of norm $d$. Then $C^{-1}$ and $D^{-1}$ exist, and $d D^{-1}$ is integral. We apply Theorem 1 and we find that (see section 3.3 of Richter [11] for details)

$$
\chi\left(\left(\begin{array}{cc}
\alpha & \beta \\
\gamma & \delta
\end{array}\right), Q\right)=\varepsilon_{d}^{-n}\left(\frac{\left(d \delta^{-1} 2 \gamma\right)^{n} \operatorname{det}(Q)}{\delta}\right),
$$

where $\varepsilon_{d}=1$ for $d \equiv 1 \bmod 4$ and $\varepsilon_{d}=i$ for $d \equiv 3 \bmod 4$ and $(\dot{\bar{\delta}})$ is the quadratic symbol over $\mathfrak{D}_{K}$.

4.2. The elliptic transformation. The elliptic transformation (18) can be proved as in the classical case (see Eichler and Zagier [2]). Let $w={ }^{t}\left(w_{1}, \ldots{ }^{t}{ }^{t} w_{r_{1}+2 r_{2}}\right)$ be as in Definition 1 and suppose further that $w_{1} \in \widetilde{J}^{n}$ such that $w_{j}=w_{1}^{(j)}$ for all $j$ and such that $Q^{(j)} w_{j}=R_{j} w_{j}$ for $j=1, \ldots, r_{1}$. Let $(\lambda, \mu) \in \mathfrak{D}_{K}^{2}$. Then it is easy to check that

$$
\begin{aligned}
\exp \{\pi i & \left.\mathscr{T}_{\mathscr{R}}[(L w)(\lambda \tau \lambda+2 \lambda z) L w]\right\} \Theta_{Q, R, w}^{(K)}((\lambda, \mu) \circ(\tau, z)) \\
= & \sum_{\iota=\left(\kappa+\lambda w_{1}\right) \in \mathfrak{J}^{n}} \exp \left\{\pi i \left(\sum_{j=1}^{r_{1}} Q^{(j)}\left[\iota^{(j)}\right] x_{j}+i R_{j}\left[\iota^{(j)}\right] y_{j}+2 \iota^{t^{(j)}} Q^{(j)} w_{j} z_{j}\right.\right. \\
& +\sum_{j=r_{1}+1}^{r_{1}+r_{2}}\left\|t^{(j)} L_{j} \tau_{j} L_{j} \iota^{(j)}+2{ }^{t_{\iota}(j)} L_{j} z_{j} L_{j} w_{j}\right\|_{\mathbb{C}} \\
& \left.\left.+\sum_{j=r_{1}+1}^{r_{1}+r_{2}}\left\|t^{(j)}{ }^{t} L_{j} \tau_{j} L_{j} \iota^{(j)}+2{ }^{t_{\iota}(j)} L_{j} z_{j} L_{j} w_{j}\right\|_{\overline{\mathbb{C}}}\right)\right\} \\
= & \Theta_{Q, R, w}^{(K)}(\tau, z),
\end{aligned}
$$

and this is the elliptic transformation (18). 


\section{An Alternate Construction}

It is well known that Jacobi forms (over $\mathbb{Q}$ ) appear as Fourier coefficients of Siegel modular forms (see Eichler and Zagier [2], and for Jacobi forms of higher degree, see Krieg [7]). In this section, we follow Skogman [12] and [13] and we present an example of an analogous situation over $K$. We show that sums of the Jacobi theta function $\Theta_{Q, R, w}^{(K)}(\tau, z)$ appear as coefficients of the symplectic theta function $\vartheta\left(Z,\left(\begin{array}{l}0 \\ 0\end{array}\right)\right)$ (roughly speaking, a Siegel modular form of weight $1 / 2$ ). Then (7) yields another proof that sums of $\Theta_{Q, R, w}^{(K)}(\tau, z)$ satisfy the transformation laws (17) and (18). Note that this method is not sufficient to prove that each theta function $\Theta_{Q, R, w}^{(K)}(\tau, z)$ satisfies $(17)$ and $(18)$.

Let $\tau, \tau^{\prime} \in \mathfrak{H}$, and $z=\left(z_{1}, \ldots, z_{r_{1}+r_{2}}\right) \in \mathscr{Z}$, i.e. $z_{j} \in \mathbb{C}$ for $j=1, \ldots, r_{1}$, and $z_{j}=u_{j}+v_{j} k \in \mathscr{Q}$ for $j=r_{1}+1, \ldots, r_{1}+r_{2}$. Let $Z^{*}$ be as in (22) and let $Z^{\prime *}$ be the matrix corresponding to $\tau^{\prime}$. For $j=1, \ldots, r_{1}$, we set

$$
\widehat{Z_{j}^{*}}=\left(\begin{array}{cc}
z_{j} I_{p} & \\
& -\bar{z}_{j} I_{q}
\end{array}\right)
$$

and for $j=r_{1}+1, \ldots, r_{1}+r_{2}$, we define the $n \times n$ matrices $\widehat{U}_{j}=u_{j} I_{n}, \widehat{\widehat{U}}_{j}=\bar{u}_{j} I_{n}$, $\widehat{V}_{j}=v_{j} I_{n}, \widehat{\widehat{V}}_{j}=\bar{v}_{j} I_{n}$. We also define the $n \operatorname{deg} K \times n \operatorname{deg} K$ matrix

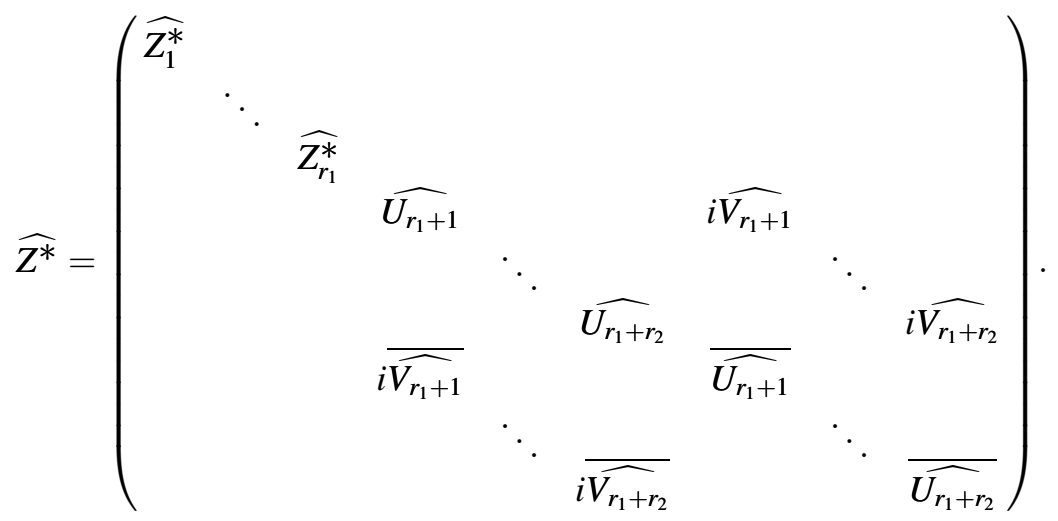

Furthermore, we define

$$
\widetilde{Z^{*}}=\left(\begin{array}{cc}
Z^{*} & \widehat{Z^{*}} \\
Z^{*} & Z^{\prime *}
\end{array}\right) .
$$

With $T$ as in (23), set

$$
\widetilde{T}=\left(\begin{array}{ll}
T & 0 \\
0 & T
\end{array}\right)
$$

and

$$
\widetilde{Z}=\widetilde{T} \widetilde{Z^{*}} \widetilde{T} .
$$

Note that $\widetilde{Z}=\overline{\widetilde{T}} \widetilde{S^{*}} \widetilde{Z^{*}} \widetilde{T}$, where

$$
\widetilde{S^{*}}=\left(\begin{array}{ll}
S^{*} & \\
& S^{*}
\end{array}\right)
$$


and

$$
S^{*}=\left(\begin{array}{ccc}
I_{n r_{1}} & & \\
& & I_{n r_{2}} \\
& I_{n r_{2}} &
\end{array}\right) .
$$

One can check that $\operatorname{Im}\left(\widetilde{S^{*}} \widetilde{Z^{*}}\right)>0$ and hence $\operatorname{Im} \widetilde{Z}>0$ and $\widetilde{Z} \in \mathfrak{H}^{(2 n \operatorname{deg} K)}$. We find that

$$
\begin{aligned}
\vartheta\left(\widetilde{Z},\left(\begin{array}{l}
0 \\
0
\end{array}\right)\right)= & \sum_{\omega \in \mathfrak{J}^{n}} \Theta_{Q, R, \Omega}^{(K)}(\tau, z) \exp \left\{\pi i \left(\sum_{j=1}^{r_{1}} Q^{(j)}\left[\omega^{(j)}\right] x_{j}^{\prime}+i R_{j}\left[\omega^{(j)}\right] y_{j}^{\prime}\right.\right. \\
& \left.\left.+\sum_{j=r_{1}+1}^{r_{1}+r_{2}} Q^{(j)}\left[\omega^{(j)}\right] x_{j}^{\prime}+\overline{Q^{(j)}}\left[\overline{\omega^{(j)}}\right] \overline{x_{j}^{\prime}}+2 i R_{j}\left\{\omega^{(j)}\right\} y_{j}^{\prime}\right)\right\},
\end{aligned}
$$

where $\Omega={ }^{t}\left(\omega^{(1)}, \ldots,{ }^{t} \omega^{\left(r_{1}+2 r_{2}\right)}\right)$.

The representation in (35) shows that the Jacobi theta functions $\Theta_{Q, R, \Omega}^{(K)}(\tau, z)$ arise as Fourier coefficients with respect to the $\tau^{\prime}$ variables. However, there may be a number of vectors $\omega$ in $\mathfrak{J}^{n}$ which are all part of the same Fourier coefficient; In some cases, $Q^{(j)}\left[\omega_{1}^{(j)}\right]=Q^{(j)}\left[\omega_{2}^{(j)}\right]$ and $R_{j}\left[\omega_{1}^{(j)}\right]=R_{j}\left[\omega_{2}^{(j)}\right]$ for all $j$, although $\omega_{1} \neq \omega_{2}$. Hence, (35) does not allow us to examine transformation properties of the single theta function $\Theta_{Q, R, \Omega}^{(K)}(\tau, z)$. In addition, we require, as in Theorem 3 , that $Q^{(j)} \omega^{(j)}=R_{j} \omega^{(j)}$ for $j=1, \ldots, r_{1}$, and we determine transformation properties for the following sums:

$$
\begin{gathered}
\Theta_{\Omega}(\tau, z)=\sum_{\kappa \in \mathfrak{J}^{n}} \Theta_{Q, R, \Omega}^{(K)}(\tau, z) . \\
Q^{(j)}\left[\omega^{(j)}\right]=Q^{(j)}\left[\kappa^{(j)}\right], 1 \leqslant j \leqslant r_{1}+2 r_{2} \\
R_{j}\left[\omega^{(j)}\right]=R_{j}\left[\kappa^{(j)}\right], 1 \leqslant j \leqslant r_{1}+2 r_{2} \\
Q^{(j)} \omega^{(j)}=R_{j} \omega^{(j)}, 1 \leqslant j \leqslant r_{1}
\end{gathered}
$$

5.1. The modular transformation. We will show that $\Theta_{\Omega}(\tau, z)$ satisfies (17). We embed $\left(\begin{array}{ll}\alpha & \beta \\ \gamma & \delta\end{array}\right) \in \Gamma$ into the symplectic group and define

$$
\left(\begin{array}{cc}
\widetilde{A} & \widetilde{B} \\
\widetilde{C} & \widetilde{D}
\end{array}\right)=\left(\begin{array}{cccc}
A & 0 & B & 0 \\
0 & I_{n} & 0 & 0 \\
C & 0 & D & 0 \\
0 & 0 & 0 & I_{n}
\end{array}\right)
$$

where $A, B, C$, and $D$ are given by (26). If

$$
\left(\begin{array}{cc}
\alpha & \beta \\
\gamma & \delta
\end{array}\right) \in \Gamma_{0}\left(\mathfrak{J}^{2} \delta_{K} \mathfrak{N}\right)
$$

we have

$$
\left(\begin{array}{cc}
\widetilde{A} & \widetilde{B} \\
\widetilde{C} & \widetilde{D}
\end{array}\right) \in \Gamma_{\vartheta}^{(2 n \operatorname{deg} K)}
$$


The symplectic transformation

$$
(\widetilde{A} \widetilde{Z}+\widetilde{B})(\widetilde{C} \widetilde{Z}+\widetilde{D})^{-1}
$$

corresponds to the transformations

$$
\begin{gathered}
(\tau, z) \longrightarrow\left(\begin{array}{ll}
\alpha & \beta \\
\gamma & \delta
\end{array}\right) \circ(\tau, z), \\
\tau_{j}^{\prime} \longrightarrow \tau_{j}^{\prime}-\frac{\gamma^{(j)} z_{j}^{2}}{\gamma^{(j)} \tau_{j}+\delta^{(j)}}, \quad \text { for } \quad 1 \leqslant j \leqslant r_{1},
\end{gathered}
$$

and

$$
\tau_{j}^{\prime} \longrightarrow \tau_{j}^{\prime}-\left(u_{j}+\overline{v_{j}} k\right)\left(\gamma^{(j)} \tau_{j}+\delta^{(j)}\right)^{-1} \gamma^{(j)} z_{j}, \quad \text { for } \quad r_{1}+1 \leqslant j \leqslant r_{1}+r_{2} .
$$

As in section 4.1, we have

$$
\operatorname{det}(C Z+D)=\mathscr{N}(\gamma \tau+\delta)^{p / 2} \mathscr{N}(\overline{\gamma \tau+\delta})^{q / 2} .
$$

The actual Fourier expansion in (35) is only with respect to the $x_{j}^{\prime}$ variables. However, it is easy to see that one can compare coefficients in (35) after applying (7) (see also [12]). We find that

$$
\begin{aligned}
\Theta_{\Omega}( & \left.\left(\begin{array}{ll}
\alpha & \beta \\
\gamma & \delta
\end{array}\right) \circ(\tau, z)\right) \\
= & \chi\left(\left(\begin{array}{ll}
\alpha & \beta \\
\gamma & \delta
\end{array}\right), Q\right) \mathscr{N}(\gamma \tau+\delta)^{p / 2} \mathscr{N}(\overline{\gamma \tau+\delta})^{q / 2} \\
& \left.\times \exp \left\{\pi i \mathscr{T}_{\mathscr{R}}^{t}(L w) z(\gamma \tau+\delta)^{-1} \gamma z L w\right]\right\} \Theta_{\Omega}(\tau, z),
\end{aligned}
$$

where $\chi\left(\left(\begin{array}{ll}\alpha & \beta \\ \gamma & \delta\end{array}\right), Q\right)$ is an eighth root of unity. Theorem 1 guarantees that $\chi\left(\left(\begin{array}{ll}\alpha & \beta \\ \gamma & \delta\end{array}\right), Q\right)$ actually coincides with the root of unity in (28).

5.2. The elliptic transformation. It remains to show that $\Theta_{\Omega}(\tau, z)$ also satisfies (18). For $(\lambda, \mu) \in \mathfrak{D}_{K}^{2}$ set

$$
\Lambda={ }^{t} T\left(\begin{array}{ccc}
\lambda^{(1)} I_{n} & & \\
& \ddots & \\
& & \lambda^{(\operatorname{deg} K)} I_{n}
\end{array}\right) T
$$

and

$$
M=T\left(\begin{array}{cccccc}
\mu^{(1)} E_{p, q} & & & & & \\
& \ddots & & & & \\
& & \mu^{\left(r_{1}\right)} E_{p, q} & & & \\
& & & \mu^{\left(r_{1}+1\right)} I_{n} & & \\
& & & & \ddots & \\
& & & & & \mu^{(\operatorname{deg} K)} I_{n}
\end{array}\right) T,
$$


where $T$ is as in (23). We embed $(\lambda, \mu)$ into the symplectic group as

$$
\left(\begin{array}{cccc}
I_{n(\operatorname{deg} K)} & 0 & 0 & M \\
\Lambda & I_{n(\operatorname{deg} K)} & M & \Lambda M \\
0 & 0 & I_{n(\operatorname{deg} K)} & -\Lambda \\
0 & 0 & 0 & I_{n(\operatorname{deg} K)}
\end{array}\right) .
$$

The symplectic transformation

$$
\left(\left(\begin{array}{cc}
I_{n(\operatorname{deg} K)} & 0 \\
\Lambda & I_{n(\operatorname{deg} K)}
\end{array}\right) \widetilde{Z}+\left(\begin{array}{cc}
0 & M \\
M & \Lambda M
\end{array}\right)\right)\left(\begin{array}{cc}
I_{n(\operatorname{deg} K)} & -\Lambda \\
0 & I_{n(\operatorname{deg} K)}
\end{array}\right)^{-1}
$$

corresponds to the transformations

$$
\begin{gathered}
\tau_{j} \longrightarrow \tau_{j}, z_{j} \longrightarrow z_{j}+\tau_{j} \lambda^{(j)}+\mu^{(j)}, \\
\tau_{j}^{\prime} \longrightarrow \tau_{j}^{\prime}+\lambda^{(j)} \tau_{j} \lambda^{(j)}+2 \lambda^{(j)} z_{j}+\lambda^{(j)} \mu^{(j)}, \forall j .
\end{gathered}
$$

We apply (7), use Theorem 1, and compare coefficients in (35) to obtain

$$
\begin{aligned}
& \Theta_{\Omega}((\lambda, \mu) \circ(\tau, z)) \\
& \left.\quad=\exp \left\{-\pi i \mathscr{T}_{\mathscr{R}}{ }^{t}(L \omega)(\lambda \tau \lambda+2 \lambda z) L \omega\right]\right\} \Theta_{\Omega}(\tau, z),
\end{aligned}
$$

which is the transformation law (18).

\section{Conclusion}

We have presented two different ways to construct examples of complex-valued functions over a number field $K$ which satisfy transformation laws generalizing those of Jacobi forms (as in Eichler and Zagier [2]) and skew-holomorphic Jacobi forms (as in Skoruppa [14]). The first method was based on Eichler's "embedding trick": We regarded $\theta_{Q, R, w, f}^{(K)}(\tau, z)$ (a certain coefficient of $\Theta_{Q, R, w}^{(K)}(\tau, z)$ ) as a specialization of $\vartheta\left(Z,\left(\begin{array}{l}r \\ s\end{array}\right), w, f\right)$, a modified version of the usual symplectic theta function $\vartheta\left(Z,\left(\begin{array}{l}r \\ s\end{array}\right)\right)$. The functional equation for $\vartheta\left(Z,\left(\begin{array}{c}r \\ s\end{array}\right), w, f\right)$ then yielded the transformation law of $\Theta_{Q, R, w}^{(K)}(\tau, z)$ under modular transformations. In the second method, we showed how sums of $\Theta_{Q, R, w}^{(K)}(\tau, z)$ appear as coefficients of $\vartheta\left(Z,\left(\begin{array}{l}0 \\ 0\end{array}\right)\right)$. This natural approach has the slight disadvantage that one can only determine transformation laws for sums of $\Theta_{Q, R, w}^{(K)}(\tau, z)$ and not for each theta function $\Theta_{Q, R, w}^{(K)}(\tau, z)$ directly.

We would like to remark that the first approach suggests a way to construct examples of vector-valued functions over $K$ which satisfy transformation properties generalizing (4) and (5). More precisely, one can define $\theta_{Q, R, w, f}^{(K)}\left(\tau, z,\left(\begin{array}{l}u \\ v\end{array}\right)\right)$ and $\Theta_{Q, R, w}^{(K)}\left(\tau, z,\left(\begin{array}{l}u \\ v\end{array}\right)\right)$, generalizations of (13) and (14) that also depend on $u=$ ${ }^{t}\left(u_{1}, \ldots,{ }^{t} u_{\operatorname{deg} K}\right)$, and $v={ }^{t}\left(v_{1}, \ldots, t_{\operatorname{deg} K}\right)$, where $u_{1}, \ldots, u_{\operatorname{deg} K}$ and $v_{1}, \ldots, v_{\operatorname{deg} K}$ are vectors in $\mathbb{C}^{n}$ (see Definition 1 of [11]). A version of our main result (Theorem 3) will also hold for $\Theta_{Q, R, w}^{(K)}\left(\tau, z,\left(\begin{array}{l}u \\ v\end{array}\right)\right)$. We omit the details since the argument follows that in section 4 with the exception that one has to consider a specialization of $\vartheta\left(Z,\left(\begin{array}{c}r \\ s\end{array}\right), w, f\right)$ for some vectors $r$ and $s$ instead of $\vartheta\left(Z,\left(\begin{array}{l}0 \\ 0\end{array}\right), w, f\right)$. One may then construct a more general class of Jacobi forms by proceeding as in [11] and applying 
the differential operators in the last section of [11] to equation (17) (of the slightly generalized version of Theorem 3). In particular, when $K$ is totally complex, one can create vector-valued Jacobi forms over $K$, i.e., real analytic functions $f$ : $\mathbb{M}_{2}^{r_{2}} \times \mathscr{2}^{r_{2}} \rightarrow \mathbb{C}^{\kappa+1}$ that satisfy transformation laws similar to (4) and (5). The only difference is that the right hand side of (4) features an extra factor of $\rho^{(\kappa)}(\gamma \tau+\delta)$, where

$$
\rho^{(\kappa)}(z)=\bigotimes_{1 \leqslant j \leqslant r_{2}} \tilde{\rho}^{(\kappa)}\left(z_{j}\right),
$$

and $\tilde{\rho}(z)^{(\kappa)}$ is the $\kappa$-fold symmetric product representation of the quaternion $z \in \mathscr{Q}$.

\section{References}

[1] Eichler M (1966) Introduction to the Theory of Algebraic Numbers and Functions. New York: Academic Press

[2] Eichler M, Zagier D (1985) The Theory of Jacobi Forms. Boston: Birkhäuser

[3] Friedberg S (1986) On theta functions associated to indefinite quadratic forms. J Number Theory 23: $255-267$

[4] Hecke E (1917) Über die $L$-Funktionen und den Dirichletschen Primzahlsatz für einen beliebigen Zahlkörper, Nachrichten der K. Gesellschaft der Wissenschaften zu Göttingen, Mathematischphysikalische Klasse, 299-318

[5] Imamoḡlu Ö (1995) The Kubota symbol for $\operatorname{Sp}(2 n, Q(i))$. J Number Theory 52: 17-34

[6] Imamoglu Ö (1995) The Kubota symbol for the symplectic group over an imaginary quadratic number field. J Reine Angew Math 462: 19-30

[7] Krieg A (1996) Jacobi forms of several variables and the Maaß space. J Number Theory 56: 242-255

[8] Richter O, On transformation laws for theta functions. Rocky Mountain J Math (to appear)

[9] Richter O (2000) Theta functions of indefinite quadratic forms over real number fields. Proc Amer Math Soc 128: 701-708

[10] Richter O (2000) Theta functions of quadratic forms over imaginary quadratic fields. Acta Arith 92: $1-9$

[11] Richter O (2002) Theta functions of harmonic coefficients over number fields. J Number Theory 95: $101-121$

[12] Skogman H (2001) Jacobi forms over imaginary quadratic number fields. Acta Arith 96: 333-350

[13] Skogman H (2001) Jacobi forms over totally real number fields. Results Math 39: 169-182

[14] Skoruppa N-P (1990) Developments in the theory of Jacobi forms. Acad Sci USSR, Inst Appl Math, Khabarovsk, 167-185

[15] Stark H (1982) On the transformation formula for the symplectic theta function and applications. J Fac Sci Univ Tokyo Sect 1A Math 29: 1-12

[16] Stark H (1988) On modular forms of weight one from real quadratic fields and theta functions. J Ramanujan Math Soc 3: 63-79

Authors' addresses: O. K. Richter, Department of Mathematics, University of North Texas, Denton, TX 76203, U.S.A., e-mail: richter@unt.edu; H. Skogman, Department of Mathematics, State University of New York at Brockport, Brockport, NY 14420, U.S.A., e-mail: hskogman@ brockport.edu 